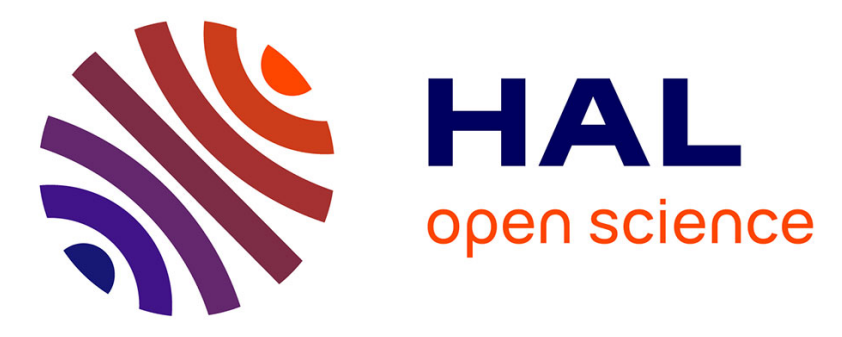

\title{
Domain decomposition approach for FEM quasistatic modeling and control of Continuum Robots with rigid vertebras
}

Julien Bosman, Thor Morales Bieze, Othman Lakhal, Mario Sanz, Rochdi Merzouki, Christian Duriez

\section{To cite this version:}

Julien Bosman, Thor Morales Bieze, Othman Lakhal, Mario Sanz, Rochdi Merzouki, et al.. Domain decomposition approach for FEM quasistatic modeling and control of Continuum Robots with rigid vertebras. IEEE International Conference on Robotics and Automation, May 2015, Seattle, United States. 10.1109/ICRA.2015.7139803 . hal-01183293

\section{HAL Id: hal-01183293 \\ https://inria.hal.science/hal-01183293}

Submitted on 9 Oct 2015

HAL is a multi-disciplinary open access archive for the deposit and dissemination of scientific research documents, whether they are published or not. The documents may come from teaching and research institutions in France or abroad, or from public or private research centers.
L'archive ouverte pluridisciplinaire HAL, est destinée au dépôt et à la diffusion de documents scientifiques de niveau recherche, publiés ou non, émanant des établissements d'enseignement et de recherche français ou étrangers, des laboratoires publics ou privés. 


\title{
Domain decomposition approach for FEM quasistatic modeling and control of Continuum Robots with Rigid Vertebras
}

\author{
Julien Bosman, Thor Morales Bieze, Othman Lakhal, Mario Sanz, Rochdi Merzouki and Christian Duriez
}

\begin{abstract}
This paper presents a development of a new method dedicated to the modeling and control of Continuum Robots, based on the Finite Element Method (FEM) using quasi-static assumption. The modeling relies on a discretization of the continuum robots using 6 DoFs Frames along the structure of the robot that is compatible with the modeling of a sequence of rigid vertebras. When the robot's structure relies on rods with constant sections, internal forces are computed with beam elements, placed between two adjacent frames, that applies forces and torques. In the opposite, when the robot is composed of a complex shape deformable backbone separated by the rigid vertebras, a domain decomposition strategy is used to obtain an equivalent stiffness between two vertebras using volumetric FEM. In both cases, for solving the whole robot model and inverting it in real-time, the numerical method takes advantage of the serial nature of continuum robots, using a Block-Tri-Diagonal solver. The factor of improvement in the computation time reaches several order of magnitude compared to a classical FEM model, while keeping a good precision. The method has also been implemented and tested on a real pneumatic CBHA trunk designed by Festo Robotics and some complementarity examples have been generated numerically.
\end{abstract}

\section{INTRODUCTION}

The study of continuum robots, as a special class of robots with deformation capabilities, aims at the conception, modeling and control of robotic mechanisms that create motion by deformation instead of using joints. Among the advantages of these robots, the production and operation of this kind of robots is cost effective compared to their classical counterpart. These ones are more robust and less dangerous, making them appealing for a large number of applications. However the modeling for the control of this type of robots is still an open problem, that we aim at (partially) addressing in this paper.

A continuum robot is a mechanism inspired by biological hydrostat muscles of elephant trunks [1], mammal tongues and tendrils [2], and octopus tentacles [3]. Such a type of robot generally consists of a backbone which supports the mechanism and an actuation system that bends or extends this backbone in order to achieve a desired posture. Among the variety of structure designs of continuum robots [4], the most common are: (1) Concentric-tube type: this robot design consists of curved concentric tubes; by varying the relative displacement and the rotation of the tubes, the robot shape is controlled; (2) Tendon actuated type: in this design, the backbone is actuated by a set of tendons or rods routed along the backbone; by pulling this set of tendons, the backbone

The authors are with INRIA, CNRS CRISTAL Laboratory, University of Lille. e-mail: julien.bosman@inria.fr, Thor.Bieze@ polytech-lille.fr, christian.duriez@inria.fr can be bent to achieve a desired posture; (3) Pneumatic actuated type: the backbone of the robot is also the actuation system, composed by pneumatic artificial muscles arranged in a parallel fashion; by means of different pressure values in each of the muscles, the robot can be coiled to achieve a desired pose.

Unlike the rigid-link robots, continuum robots are characterized by the absence of discrete parts, and many degrees of freedom, but in case (2) and (3), the robot contains a sequence of rigid vertebras along the principal axis of the robot. Theoretically speaking, continuum robots have infinite kinematic degrees and their natural compliance makes them suited for a large number of applications such as search and rescue operations, underwater and space operations, and medical surgery operations [5], to name a few.

In order to exploit the full capabilities of a continuum robot, a model that relates the external forces applied to the robot and the resultant posture of the continuum backbone is required. This paper seeks to illustrate a new modeling approach based on finite element method (FEM) to determine the shape of the continuum by taking into account the internal forces present in the robot and the relation of these forces to the deformation of the structure. Precise FEM models are, in general, very time consuming and unadapted to be used in the control loop of the robot. However, our previous work [6] shows that real-time implementation of volume FEM can be used for modeling soft-robot. However the real-time constraint limits the maximum size of admissible meshes and the refresh-rate of the model is still quite low.

In this paper, we focus on continuum robots that are often made of a slender structure. Instead of discretizing of the whole volume of the soft robot structure, we propose to rely on a sampling of the main axis deformation and benefit from a very efficient Block-Tri-Diagonal (BTD) solver to compute the inverse model. In order to capture the possible complex shape of the backbone between the vertebras (especially in the case (3) cited above), we rely on a two steps sequence of substructure analysis: First, a highly detailed FEM model is computed offline, for each intervertebra, to obtain, with domain decomposition, an equivalent stiffness between two vertebras. Second, a real-time corotational approach is used to compute the deformations of the whole structure and compute the inverse model using the BTD solver. Finally, some numerical examples are presented in the simulation, and we have conducted an experiment on a special class of continuum robot based on intrinsic pneumatic actuation: the Compact Bionic Handling Assistant designed by Festo Robotics. 


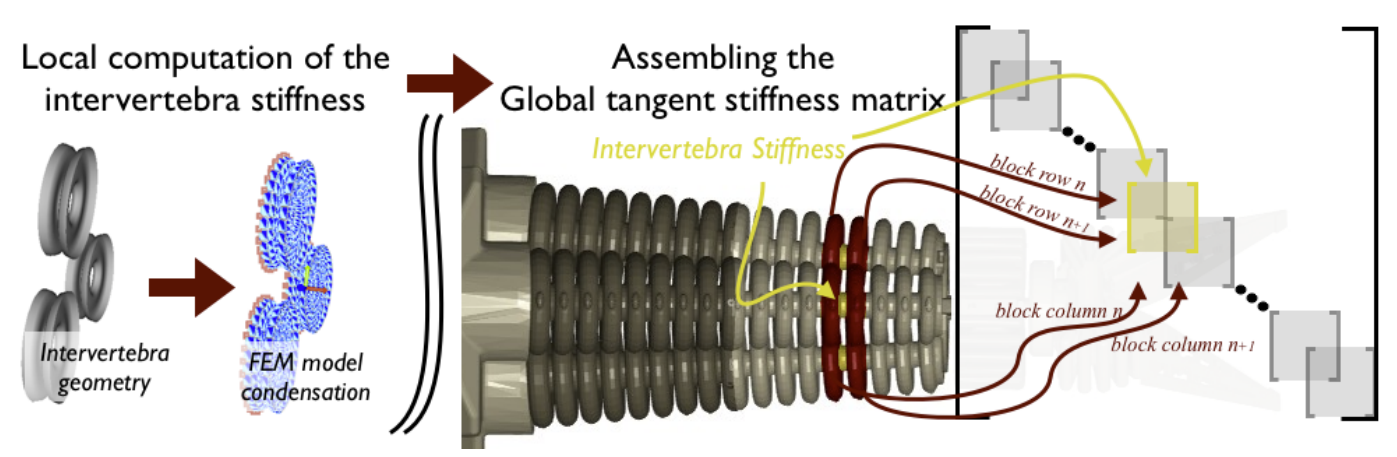

Fig. 1: Left: Domain decomposition strategy to extract the equivalent local stiffness between frames. Right: Assembling various local stiffnesses leads to a Block Tri Diagonal system that can be solved very efficiently in real-time even for large matrices.

\section{RELATED WORK}

The modeling and control of continuum robots is challenging owing to their large number of unactuated degrees of freedom, as well as the implicit compliance of the mechanism to external contact forces and gravity. The elasticity of the backbone plays an important role in the determination of the final posture of the robot. The assumption of piecewise constant curvature has been used to represent the backbone of continuum robots [7] [8]; this assumption results in low fidelity models that provide a simple determination of the kinematics of the robot at the cost of accuracy in the results. This is due to external loading and gravity effects that can cause some sagging in the flexible backbone.

Although the kinematic models of continuum manipulator robots are currently well developed, accurate dynamic models of continuum robots are a necessity as the applications for such mechanisms become more complex. Taking internal and external forces acting on the robots into account has become a minimum requirement in every dynamic model. In [9], Gravagne et al. propose a continuum mechanics based approach to compute the dynamics of a highly deformable planar backbone.

\section{DOMAIN DECOMPOSITION APPROACH}

One of the main features of the modeling presented in this paper is to discretize the deformation of continuum robots using a set of frames (which are nodes with 6 degrees of freedom (DoF) nodes : 3 in translation and 3 in rotation, like rigid bodies) aligned along the principal axis of the robot. The position $\mathbf{q}$ of the robot is then given by gathering the DoFs defined at each nodes. In between these frames, we compute a non-linear stiffness $\mathbf{f}(\mathbf{q})$ that is derived from physics based modeling of continuum mechanics.

When the robotic arm has a uniform cross section like some of the tendon actuated continuum robots, we use the beam elements [10] extended to large rotations in [11] to compute the internal forces (see Fig. 6). However, in the case of more complex shapes, the use of surface (shell) or even volume elements becomes necessary to faithfully reproduce their behavior. This approach allows for a more precise control of continuum robots but it puts a hard constraint on the performance, because of the large increase in the number of degrees of freedom. Yet, computation times must be kept as low as possible to enable real-time interaction and smooth motion of the robot. Nevertheless, this constraint is hardly compatible with a precise FEM simulation and inverse model, mainly because of the high number of elements required and its computational cost.

Still, one can note that continuum robotic arms with rigid vertebras are made of the same repeated substructure. Thus, a given part can be isolated and its mechanical properties computed. These mechanical properties can then be used to substitute the FEM simulation for a computationally more efficient technique allowing for high computation rate.

One of the main contribution of this work is to use a condensation of a full FE model on the frames defined on the main axis of the robot. To obtain the same mechanical behavior, the equivalent stiffness of the $\mathrm{FE}$ model must be computed to be transfered on the springs between two consecutive frames. On the performance side, the equivalent stiffness matrix can be precomputed which allows to perform most of the computation off-line. One interesting characteristic of this technique, inspired by substructure analysis, is that it relies on the use of mixed node types, i.e. classical 3 DoF finite elements nodes and frames.

To precompute the equivalent stiffness matrix, the simulation is organized as follows: a subpart of the robotic arm is isolated and modeled using FEM, and two frames are placed in the middle at both upper and lower ends of the subpart (Fig. 2). The role of the frame is to simulate a rigid link between subparts. Hence, FE nodes at both ends of the subpart are kinematically linked to their respective frame, and have a pure rigid motion, while the other FE nodes have a independent motion. In a similar manner, the motion of the frames is influenced by the FE nodes they are linked to.

The first step in the computation of the equivalent stiffness matrix is to compute the stiffness matrix of a subpart, taking the influence of the frames into account. As a consequence, FE nodes and frames have to be merged into the same mechanical system since both upper and lower boundary nodes are reduced to a frame.

Using substructure analysis techniques such as in [10] 


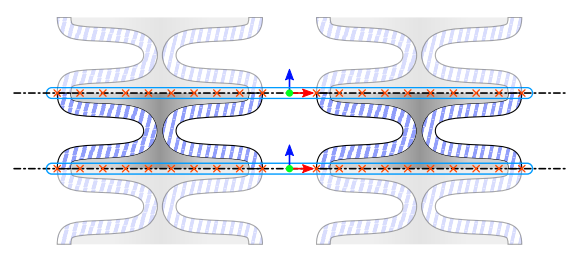

Fig. 2: Substructure used for the computations. The red crosses indicate the FE nodes that are kinematically linked to the frames.

( 9.1$)$, the stiffness matrix of the substructure can be decomposed in four blocks:

$$
\mathbf{K}=\left[\begin{array}{ll}
\mathbf{K}_{I I} & \mathbf{K}_{I R} \\
\mathbf{K}_{R I} & \mathbf{K}_{R R}
\end{array}\right]
$$

where the off-diagonal blocks describe the influence of the frames on the FE nodes $\left(\mathbf{K}_{R I}\right)$ and conversely $\left(\mathbf{K}_{I R}\right)$, due to the kinematic rigidity imposed by the frames.

Once the $\mathbf{K}$ matrix is computed, it can be factorized and inverted to obtain the compliance matrix for both FE nodes and frames.

The next step consists in the extraction of the frames related part of the compliance matrix. In this work, the compliance matrix needed to build the equivalent stiffness of frames is computed using a constraint based approach: the bottom frame is fixed, and a unit force and torque are applied successively in each direction on the upper frame. It results in 6 constraints for the upper frame (one constraint per DoF). As a result, one obtains a deformation measure of the substructure, under a unit load in each direction, which is equivalent to the compliance matrix ${ }^{1}$ :

$$
\mathbf{C}=\mathbf{J K}^{-1} \mathbf{J}^{t}
$$

where

$$
\mathbf{J}=\left[\begin{array}{ll}
\mathbf{0}^{6 \times i} & \mathbf{I}^{6 \times 6}
\end{array}\right]
$$

with $\mathbf{0}^{m \times n}$ being a zero matrix, $i$ the number of independent FE nodes, and the $6 \times 6$ identity matrix corresponds to the constraints on each DoF of the upper frame.

The $\mathbf{C}$ compliance matrix enables for both frames force and equivalent stiffness matrix computations. Let $\mathbf{q}_{0}=\left[\mathbf{x}_{0} \mathbf{r}_{0}\right]$ and $\mathbf{q}_{1}=\left[\mathbf{x}_{1} \mathbf{r}_{1}\right]$ the lower and upper frames with their respective position and orientation (represented by quaternions in practice). In order to compute a reaction force, displacements must be evaluated. These latter are expressed in the $\mathbf{q}_{0}$ coordinate system. The force acting on $\mathbf{q}_{1}$ in $\mathbf{q}_{0}$ local coordinates, noted $\mathbf{f}_{1 / 0}$, can be computed using the compliance:

$$
\mathbf{f}_{1 / 0}=\mathbf{C}_{0,1}^{-1} \partial \mathbf{q}_{1 / 0}
$$

where $\partial \mathbf{q}_{1 / 0}$ is the $\mathbf{q}_{1}$ displacement in the $\mathbf{q}_{0}$ coordinate system and $\mathbf{C}_{0,1}$ is the compliance between frames 0 and 1 . Then, using $\mathbf{f}_{1 / 0}$, force and torque can be applied on $\mathbf{q}_{0}$ :

$$
\mathbf{f}_{0 / 0}=\mathbf{H}_{0,1} \mathbf{f}_{1 / 0}
$$

\footnotetext{
${ }^{1}$ In practice $\mathbf{K}$ is not inverted but only factorized to keep a sparse format. $\mathbf{C}$ is then pre-computed by system solving in a few seconds
}

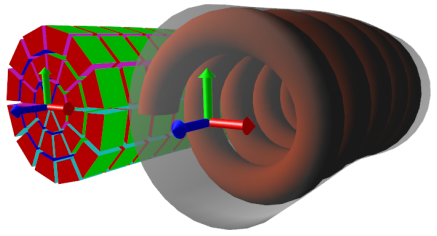

Fig. 3: Comparison between two cylinders simulated using FEM on the left and equivalent stiffness method on the right.

with

$$
\mathbf{H}_{0,1}=-\left[\begin{array}{cc}
\mathbf{I}^{3 \times 3} & \mathbf{0}^{3 \times 3} \\
\left(\mathbf{x}_{1}-\mathbf{x}_{0}\right)^{\wedge} & \mathbf{I}^{3 \times 3}
\end{array}\right]
$$

describing the relationship between linear displacement on frame 1 and angular displacement on frame 0 , where $\mathbf{v}^{\wedge}$ is a skew-symmetric matrix obtained from the $\mathbf{v}$ vector. Finally, forces in global coordinates can be obtained by:

$$
\mathbf{f}_{0}=\mathbf{R}_{0} \mathbf{f}_{0 / 0}, \mathbf{f}_{1}=\mathbf{R}_{0} \mathbf{f}_{1 / 0}
$$

With $\mathbf{R}_{i}$ being the rotation matrix of the frame $i$. The equivalent stiffness matrix can be computed alike. For instance, given two consecutive frames $i$ and $j$ the equivalent stiffness matrix can be expressed as :

$$
\mathbf{K}_{i, j}^{e q}=\mathbf{R}_{i}\left[\begin{array}{cc}
\mathbf{H}_{i, j} \mathbf{C}_{i, j}^{-1} \mathbf{H}_{i, j}^{t} & \mathbf{H}_{i, j} \mathbf{C}_{i, j}^{-1} \\
\mathbf{C}_{i, j}^{-1} \mathbf{H}_{i, j}^{t} & \mathbf{C}_{i, j}^{-1}
\end{array}\right] \mathbf{R}_{i}^{t}
$$

Using this approach, the FE model of a whole robotic arm can be replaced by a linear structure similar to a chain made of frames linked by equivalent springs. In this case, all the frames, except the first and the last one, are linked to exactly two adjacent frames. As a consequence, the global stiffness matrix assembly process highlights an interesting property of the method, as illustrated in Fig. 1. The resulting global stiffness matrix is block tridiagonal which allows for the use of optimized resolution techniques [12]. One can note that it is possible to use different compliance matrices which is another advantage of this work. It implies that this method allows to reproduce a robotic arm made of different kind of subparts, as long as their compliance matrix is provided.

In order to validate the method, some tests where made as show on the Fig. 3. The difference between the FE cylinder and its equivalent frame model is inferior to $0.72 \%$.

\section{Control By inVerse Simulation}

This work is built on [6], which proposes to model the non-linear deformation of volumetric soft robots - using the Finite Element Method - at low refresh rates, and using a non-standard optimization solver. Starting from the quasi-static formulation of the equilibrium between internal forces $\mathbf{f}(\mathbf{q})$, described above, external loads $\mathbf{f}_{\text {ext }}$ (like the gravity) and contributions of the actuators $\mathbf{J}^{T} \lambda_{a}$ (where $\mathbf{J}^{T}$ is a jacobian matrix described below and $\lambda_{a}$ is the unknown contribution of each actuator. As a result the equilibrium formulation for the entire robot is given by:

$$
\mathbf{f}(\mathbf{q})+\mathbf{f}_{e x t}+\mathbf{J}^{T} \lambda_{a}=0
$$


In our study, two models of actuations are introduced. The first one corresponds to tendon actuator: the continuum robot is driven by cables. These cables pass through the vertebras and apply some forces that are transported to the frame using a rigid mapping assumption (see Fig. 4). We also consider that there is no friction between the tendons and the vertebras. In that case, for each cable, the jacobian $\mathbf{J}^{T}$ contains a column that provides the weighted direction of the force exerted by the cable on each frame. In other words, when multiplying this column by the unknown intensity of the force $\lambda_{a}$ exerted by the actuator on the cable, it provides the forces exerted by the cable on the frames.

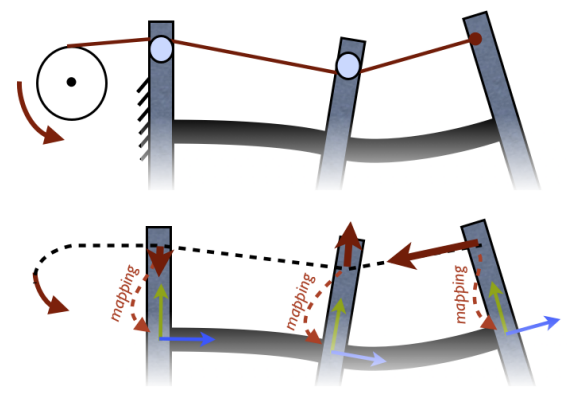

Fig. 4: Cable actuator. The constraint jacobian is given by the reaction forces direction created on the structure when each cable is being pulled and then mapped on the frames.

The second one corresponds to pressure actuation. Some deformable cavities are placed between the vertebras (or on several vertebras as it is the case with the CBHA) that can be inflated by change of pressure. The Domain decomposition approach allows to account, at the frame level, for the internal forces created by the deformation of the cavities. However,

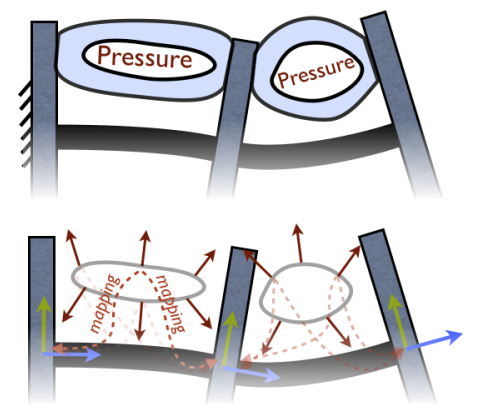

Fig. 5: Pressure actuator. The constraint jacobian is build using the forces on the points of the cavity created by unity of pressure ; these forces are then mapped back to the frame using skinning mapping.

the pressure exerted on the cavities will create additional forces: at each point of the cavity model (composed of a triangle mesh), we take the direction of the surface and multiply it by the area of the adjacent triangles divided by 3. This way, we can have a weighted direction of force that we map at the frame level using the transposed jacobian of a skinning mapping [13]. In the resulting jacobian $\mathbf{J}^{T}$, each column contains the weighted directions of the forces exerted on each frame by a unity of pressure in a cavity. In that case $\lambda_{a}$ represents the unknown pressure in the cavity, that have to be found by control.

We also define some additional constraints at the tip of the effector, using a jacobian matrix $\mathbf{J}_{e}$ that consists in a matrix filled with 0 values except on the rows that corresponds to the last frame of the robot model (the end effector), where we put an identity matrix. The goal of this jacobian is to obtain the mechanical coupling between effectors and actuators for the inverse model optimization. Moreover, if there is a prescribed force on the effector, we can set it into $\lambda_{e}$ so that $\mathbf{J}_{e}^{T} \lambda_{e}$ can be added in $\mathbf{f}_{\text {ext }}$ as an external force.

At this point we reused the method described in [6] to compute the inverse model of the robot: how much effort needs to be applied on each actuator so that it creates a deformation that corresponds to the desired motion at the end effector of the soft-robot.

First, we use the a linearization of the internal forces exerted on the frames at each step $i$ of the computation:

$$
\mathbf{f}\left(\mathbf{q}_{\mathbf{i}}\right) \approx \mathbf{f}\left(\mathbf{q}_{\mathbf{i}-\mathbf{1}}\right)+\mathbf{K}\left(\mathbf{q}_{\mathbf{i}-\mathbf{1}}\right) d \mathbf{q}
$$

where $\mathbf{K}\left(\mathbf{q}_{\mathbf{i}-\mathbf{1}}\right)$ is a Block Tridiagonal matrix, as described above.

Then, we project the mechanics into the constraint space using the Shur complement of the constraint problem:

$$
\begin{aligned}
& \delta_{e}=\underbrace{\left[\mathbf{J}_{e} \mathbf{K}^{-1} \mathbf{J}_{a}^{T}\right]}_{\mathbf{W}_{e a}} \lambda_{a}+\delta_{e}^{\text {free }} \\
& \delta_{a}=\underbrace{\left[\mathbf{J}_{a} \mathbf{K}^{-1} \mathbf{J}_{a}^{T}\right]}_{\mathbf{W}_{a a}} \lambda_{a}+\delta_{a}^{\text {free }}
\end{aligned}
$$

Where $\delta_{e}^{\text {free }}$ and $\delta_{a}^{\text {free }}$ are, respectively, the gap between the effector position and the desired position $p_{d e s}$, and the displacement of the actuators when the effort of the actuator vanishes $\lambda_{a}=0$. Matrices $\mathbf{W}_{e a}$ and $\mathbf{W}_{a a}$ are homogeneous to a compliance. Using $\mathbf{W}_{e a}$, we can get a measure of the mechanical coupling between effector and actuator, and with $\mathbf{W}_{a a}$, the coupling between actuators.

Obtaining these coupling matrices $\mathbf{W}_{e a}$ and $\mathbf{W}_{a a}$ is computationally expensive because it involves the inverse of the matrix $\mathbf{K}$ issued from the FEM model. However, in the particular case of this work, matrix $\mathbf{K}$ is condensed on the frame nodes that are sampled along the axis of the robot and this matrix is block-tri-diagonal. The complexity of the factorization of such matrix is linear w.r.t. the number of frames. The computation is particularly fast, even for a large number of nodes and actuators, making the scalability of the whole approach more appealing than what was proposed in [6]. The remaining of the algorithm stays similar to [6]: a Gauss-Seidel solver is used to compute an optimization problem. It minimizes the norm of $\delta_{e}$ which measures the shift between the end effector and its desired position. It can also be seen as a quadratic minimization problem:

$$
\min \left(\frac{1}{2} \lambda_{a}{ }^{T} \mathbf{W}_{e a}^{T} \mathbf{W}_{e a} \lambda_{a}+\lambda_{a}{ }^{T} \mathbf{W}_{e a}^{T} \delta_{e}^{\text {free }}\right)
$$


subject to the constraints defined by each actuator (unilateral actuator for cables, limited pressure values for pneumatic actuators).

When multiple solutions are possible (like in the case of the tendon actuated robot presented in the Result section), the matrix $\mathbf{W}_{e a}^{T} \mathbf{W}_{e a}$ is positive but only semi-definite, which limits the kind of minimization algorithm that can be used. This is one of the good property of the Gauss-Seidel solvers that are able to find one solution among all the possible.

\section{RESUlts}

a) Simulated example: First, we made some numerical experiments on a tendon actuated continuum robot to evaluate the scalability of the approach. The mechanical model of the robot is composed of a series of beam elements, as we consider that the section is constant in between the vertebras (see figure 6 (a) ). We have created simple examples of artificial robots made of 4 and 8 tendons with 3 mobile vertebras. The model is composed of 6 beams and 6 mobile frames ( 2 beam elements for modeling the deformation of each intervertebra) We verify that the method is able to solve the inverse problem, at very fast updates: the computation time for each step is about $5 m s$ with about $2 m s$ to compute the compliance values $\mathbf{W}_{a a}$ and $\mathbf{W}_{e a}$ for the example illustrated in figure 6. The optimization takes less than $1 m s(\mathrm{~b})^{2}$

Then, we have created a more complex scenario (see figure 6(c) with a simulated robot made of 5 mobile vertebras and actuated with 16 tendons. The model is composed of 10 beam elements and 10 frame nodes. The computation time is about $13 m s$ with $9 m s$ for computing the compliance matrices. (if we keep only 8 tendons, the computation of the compliance matrices falls to a little bit more than $4 m s$ which is about 2 times the computation time for the same number of tendons with 6 frame nodes)

On this scenario, we can optimize the trajectory for two effector points: the first is placed on the second vertebra and the second at the tip of the robot. The optimization is performed in a global manner, by trying to reduce the distance for both effector points. Given the number of actuators, the system is hyper-redundant but the Gauss-Seidel algorithm used to performed the optimization founds smooth trajectories. As we optimize the actuation for two effector positions, we can control the posture of the robot, not only the tip position.

The simulations were performed using the SOFA framework (http://www. sofa-framework.org/).

\footnotetext{
${ }^{2}$ These numerical experiments were conducted with a PC based on a 2.66 $\mathrm{GHz}$ Intel Core i7, 8Go.
}
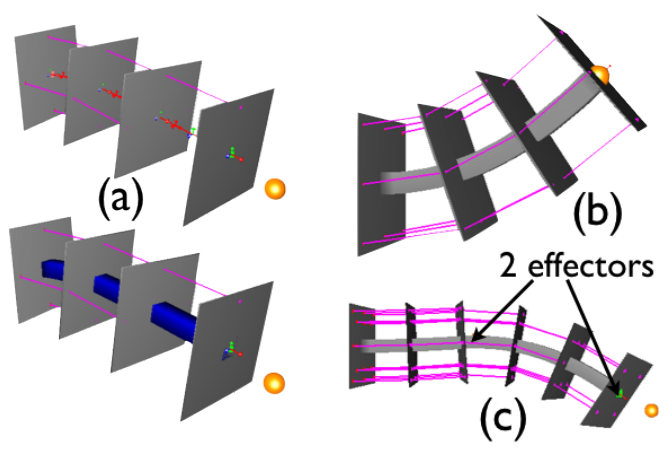

Fig. 6: Simulated example of a trunk modeled by beam elements coupled with rigid vertebras.

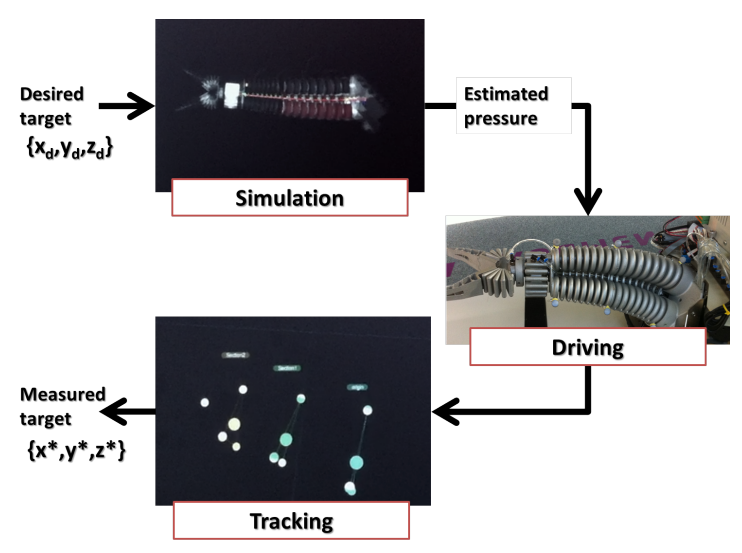

Fig. 7: Experimental setup

b) Description of the CBHA: The Compact Bionic Handling Assistant is a continuum manipulator robot inspired on the form and functionality of an elephant trunk, designed by Festo Robotics. The CBHA is part of the didactic platform named RobotinoXT; this robot is composed by an omnidirectional mobile platform and the CBHA itself. The CBHA is composed by two serially connected sections of pneumatic artificial muscles: lower and upper section; a rotative wrist and a soft gripper.

Each section is formed by three muscles arranged in parallel configuration. If differential (equal) pressures are applied to the muscles, the section can bend (extend) to change the position and orientation of its distal vertebra. The shape of the manipulator is determined by the net change in position and orientation of both sections that can be computed with the aid of string potentiometers attached to the sides of the muscles. Each muscle resembles a vertebral column or backbone, in which 8 vertebras are connected to each other serially by an inter-vertebras sections which are more flexible in comparison to the vertebras. The whole structure is composed by 16 vertebras.

c) Experimental Results: The inverse model of the robot has been tested in open-loop to control the movements of the CBHA; a desired trajectory of the tip of the manipulator is selected and the simulation computes the pressures needed in each actuator to create the required deformation 


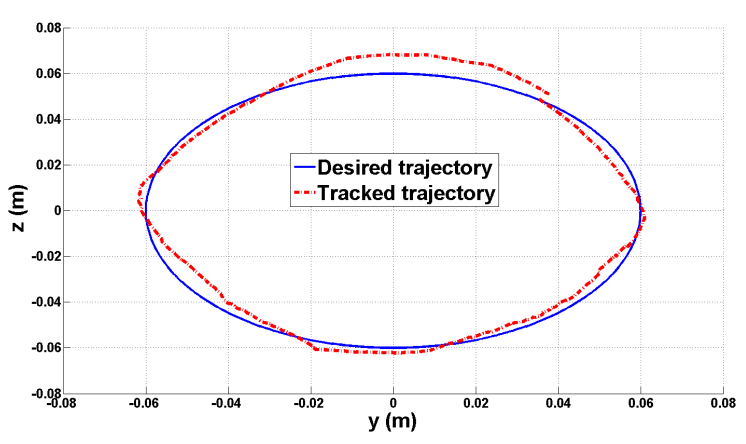

Fig. 8: Difference between tracked and desired trajectories for circle of $60 \mathrm{~mm}$ of radius

to reach the target.

A stereo-vision system was used to track the position of the tip of the arm to validate the accuracy of the model. Such setup is showed in Fig. 7 . A circle and a square in the $y-z$ plane were used as desired trajectories for the experiment. In Fig 8 and 9 the comparison between the desired and tracked trajectories is presented. In the first trajectory, the circle is selected to have a radius of $60 \mathrm{~mm}$, while a square of side lenght of $80 \mathrm{~mm}$ is selected for the second trajectory. The speed at which the square and circle trajectories are performed are $3.1 \mathrm{~cm}$ and $2.5 \mathrm{~cm}$ per second respectively, which is slow enough so that the dynamic effects can be neglected in the model. During the experiment, the estimated pressures are sent to the robot at the end of each simulation step, this causes a latency of $54 \mathrm{~ms}$ between the model and the CBHA, moreover, the pressure valves of the CBHA have a response time of $10 \mathrm{~ms}$ and the connection between the simulation and the robot has a latency of $3 \mathrm{~ms}$. It is important to notice that the results obtained during the

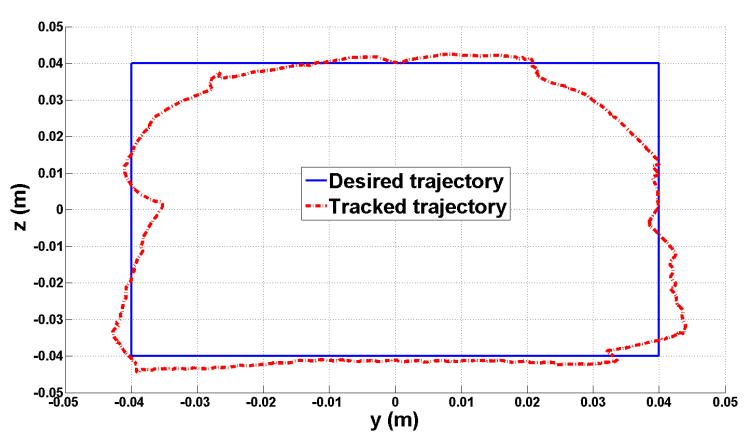

Fig. 9: Difference between tracked and desired trajectories for square of side lenght of $80 \mathrm{~mm}$

experimentation rely only on the inverse model computation. The good experimental results obtained in Figure 8 and 9 demonstrate the validity of the approach presented in this paper.

\section{CONCLUSION}

In this paper, the derivation of a method to compute the inverse model for continuum robots with diverse backbone geometry is presented. The potential and scalability of this method is demonstrated with numerical and real-time simulation experiments.

This work is a very good starting point to explore new modeling and control strategies for continuum robots. Moreover, the genericity of the approach makes it perfectly suited for new design concept with hybrid actuation (tendon and pneumatic for instance)

\section{ACKNOWLEDGMENTS}

The authors would like to thank Eulalie Coevoet for her valuable efforts in programming the solver used in the simulations.

\section{REFERENCES}

[1] S. Neppalli and B. A. Jones, "Design, construction, and analysis of a continuum robot," in 2007 IEEE/RSJ International Conference on Intelligent Robots and Systems. Ieee, Oct. 2007, pp. 1503-1507.

[2] I. D. Walker, "Robot strings: Long, thin continuum robots," in 2013 IEEE Aerospace Conference. Ieee, Mar. 2013, pp. 1-12.

[3] D. Cojocaru and M. Ivanescu, "Experiments with Tentacle Robots," in Robotics (ISR), 2010 41st International Symposium on and 2010 6th German Conference on Robotics (ROBOTIK), 2010, pp. 948-953.

[4] I. D. Walker, "Continuous Backbone "Continuum" Robot Manipulators," ISRN Robotics, vol. 2013, pp. 1-19, 2013.

[5] D. Trivedi, C. D. Rahn, W. M. Kier, and I. D. Walker, "Soft robotics: Biological inspiration, state of the art, and future research," Applied Bionics and Biomechanics, vol. 5, no. 3, pp. 99-117, Dec. 2008.

[6] C. Duriez, "Control of elastic soft robots based on real-time finite element method," in Robotics and Automation (ICRA), 2013 IEEE International Conference on. IEEE, 2013, pp. 3982-3987.

[7] R. J. Webster and B. A. Jones, "Design and Kinematic Modeling of Constant Curvature Continuum Robots : A Review," The International Journal of Robotics Research, pp. 1-56, 2010.

[8] B. A. Jones and I. Walker, "Kinematics for multisection continuum robots," IEEE Transactions on Robotics, vol. 22, no. 1, pp. 43-55, Feb. 2006.

[9] I. Gravagne, C. Rahn, and I. Walker, "Large deflection dynamics and control for planar continuum robots," Mechatronics, IEEE/ASME Transactions on, vol. 8, no. 2, pp. 299-307, June 2003.

[10] J. Przemieniecki, Theory of Matrix Structural Analysis, ser. Dover Civil and Mechanical Engineering. Dover, 1985.

[11] C. Duriez, S. Cotin, J. Lenoir, and P. F. Neumann, "New approaches to catheter navigation for interventional radiology simulation," Computer Aided Surgery, vol. 11, pp. 300-308, 2006.

[12] S. D. Conte and C. W. D. Boor, Elementary Numerical Analysis: An Algorithmic Approach, 3rd ed. McGraw-Hill Higher Education, 1980.

[13] X. C. Wang and C. Phillips, "Multi-weight enveloping: least-squares approximation techniques for skin animation," in Proceedings of the 2002 Symposium on Computer Animation. New York, NY, USA: ACM, 2002, pp. 129-138. 